\title{
Is a Nicotinic Influence Involved in Denervation-induced Depolarization of Muscle? ${ }^{1}$
}

\author{
S. ROCHEL ${ }^{2}$ AND N. ROBBINS \\ Department of Developmental Genetics and Anatomy, Case Western Reserve University, School of Medicine, \\ Cleveland, Ohio 44106
}

\begin{abstract}
The neurotrophic role of acetylcholine (ACh) in the denervation-dependent decline of muscle resting membrane potential (RMP) was evaluated. Freshly dissected rat hemidiaphragms with short or long $(2 \mathrm{~cm})$ nerve stumps attached (" $-N$ " and " $+N$ " preparations, respectively) were incubated in organ culture in the presence or absence of the nicotinic blockers, $\alpha$-bungarotoxin ( $\alpha$-BTX) or $d$-tubocurarine (curare). Subsequently, RMPs and miniature endplate potentials (MEPPs) of the junctional region were measured.

Spontaneous MEPPs disappeared with a half-life of 12 and $20 \mathrm{hr}$ in $-\mathrm{N}$ and $+\mathrm{N}$ preparations, respectively. A 10- to 15$\mathrm{mV}$ depolarization of RMP was observed between 15 and 20 $\mathrm{hr}$ in $-\mathrm{N}$ muscles and between 24 and $28 \mathrm{hr}$ in $+\mathrm{N}$ muscles. This time course of disappearance of spontaneous potentials and of membrane depolarization agrees well with that observed in vivo.
\end{abstract}

Although nicotinic transmission was blocked from the initiation of the incubation period in $\alpha$-BTX- or curare-treated muscles, no acceleration of RMP decline in $-N$ muscles in vitro was observed. Moreover, in $+N$ preparations the effect of the nerve stump in delaying RMP depolarization persisted despite the continuous presence of $\alpha$-BTX or curare.

Since excess ACh triggers a lysosomal proteolytic response at the nerve-muscle junction and since this may occur early in denervation, the possible role of a nicotinic-induced proteolytic mechanism was tested in vitro with the potent protease inhibitor leupeptin. This inhibitor did not delay or prevent the denervation-dependent alterations.

Thus, with respect to neuronal regulation of RMP, neither the effects of denervation nor of the long nerve stump are entirely explicable by a nicotinic mechanism, or by a leupeptin-sensitive proteolytic mechanism.

Denervated skeletal muscle undergoes numerous morphological and electrophysiological alterations (see reviews by Harris, 1974; Gutmann, 1976; Purves, 1976; Fambrough, 1979). The earliest postdenervation physiological events are the instant cessation of nerveevoked action potential and muscle activity, followed by failure of miniature endplate potentials (MEPPs) at about $12 \mathrm{hr}$ (Miledi and Slatcr, 1970; Card, 1977) and a decline of the muscle resting

Received June 4, 1984; Revised Feburary 8, 1985; Accepted March 4, 1985

\footnotetext{
${ }^{1}$ This work was supported by National Institutes of Health Grants NS 07118 and NS 18694 and a National Amyotrophic Lateral Sclerosis Foundation grant to $S$. R. We wish to thank Diana Guegel for technical help.

${ }^{2}$ To whom correspondence should be addressed.
}

membrane potential (RMP) at about 20 to $24 \mathrm{hr}$ (Slater, 1966; Redfern and Thesleff, 1971; Card, 1977; Drachman et al., 1982), or possibly earlier (Albuquerque et al., 1971).

Although activity plays a role in neuronal regulation of various muscle properties (Drachman and Witzke, 1972; Cohen and Fishbach, 1973; Shainberg and Burstein, 1976; Hall and Reiness, 1977; Betz and Changuex, 1979), several observations have given rise to the hypothesis that a neurotrophic influence prevents or delays denervation-induced alterations, and are consistent with the suggestion that cholinergic transmission provides the above neurotrophic influence. First, both spontaneous quantal and non-quantal acetylcholine (ACh) release decline before RMP begins to depolarize (cf. references above, and Linden et al., 1983). Second, increasing the length of the nerve stump delays the denervation-dependent failure of ncuromuscular transmission, the time of failure of spontancous quantal $A C h$ release, the loss of nerve terminal $A C h$ and $A C h$ synthesis (Miledi et al., 1981, 1982) and the depolarization of RMP (Miledi and Slater, 1970). The production of denervation characteristics when ACh receptors are blocked by $\alpha$-bungarotoxin ( $\alpha$-BTX) further supports the idea of a cholinergic involvement (Drachman et al., 1982).

The objective of the present study was to evaluate the neurotrophic role of nicotinic ACh transmission in the development of the denervation-dependent depolarization of the muscle resting membrane. If spontaneous (quantal or non-quantal) or action potentialevoked ACh release by the motor nerve were the major or sole requirement for RMP maintenance, then the denervation-induced depolarization of RMP should be accelerated by the "instant denervation" induced by nicotinic blockers. An in vivo test of this prediction would not be decisive, since nicotinic receptor blockers are reversible and difficult to regulate in vivo (e.g., by d-tubocurarine) or are relatively short-lived in functional effect. For instance, transmission block induced by in vivo $\alpha$-BTX lasts as little as $12 \mathrm{hr}$ because the recovery of only $26 \%$ of unbound receptors is enough to support impulse transmission (Rochel and Robbins, 1984). Therefore, in order to ensure that complete and continuous block of nicotinic transmission is maintained during the critical period, an in vitro system, in which several denervation phenomena occur approximately "on schedule," was used. Furthermore, emphasis was placed on the first $27 \mathrm{hr}$ after denervation, since in this and in previous reports, loss of ACh release as well as onset of membrane depolarization occurred within this period. In this system, the findings provided evidence against the cholinergic hypothesis.

Acetylcholine in high concentrations induces a leupeptin-sensitive proteolysis at the endplate region by a nicotinic mechanism (Leonard and Salpeter, 1979). In addition, the proteolytic inhibitor, leupeptin, was previously shown to prevent two denervation phenomenamuscle atrophy (Libby and Goldberg, 1980) and decrease of acetylcholinesterase (Fernandez and Duell, 1980; Cater and Brimijoin, 1981). Given reports of a transient increase in spontaneous ACh release just before failure of the denervated nerve terminal (e.g., 

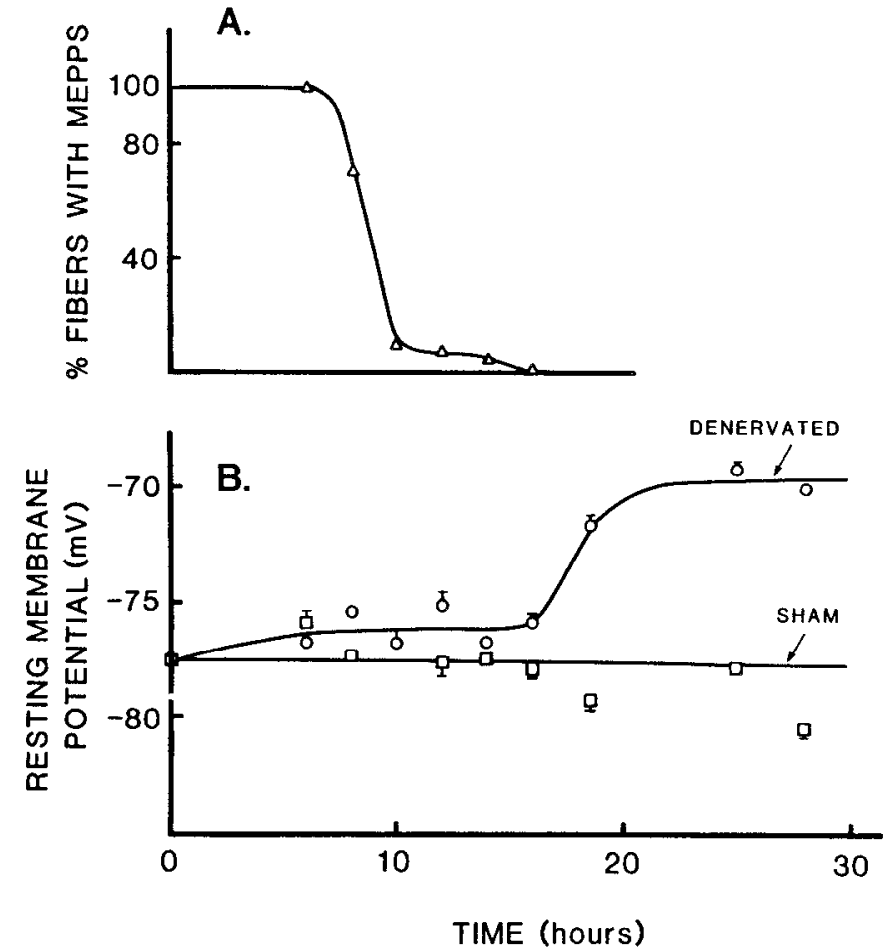

Figure 1. MEPPs and RMP after in vivo denervation. The right branch of the left phrenic nerve was sectioned, while the left branch was kept intact as an internal sham control. MEPP failure $(A)$ and resting potential $(B)$ of denervated $(O)$ and sham $(\square)$ muscles are presented as a function of time after denervation. The length of the nerve within the muscle was between 2 and $6 \mathrm{~mm}$ from the site of nerve section. Each data point represents the mean of 60 fibers from two or three muscles. Bars are standard error of the mean.

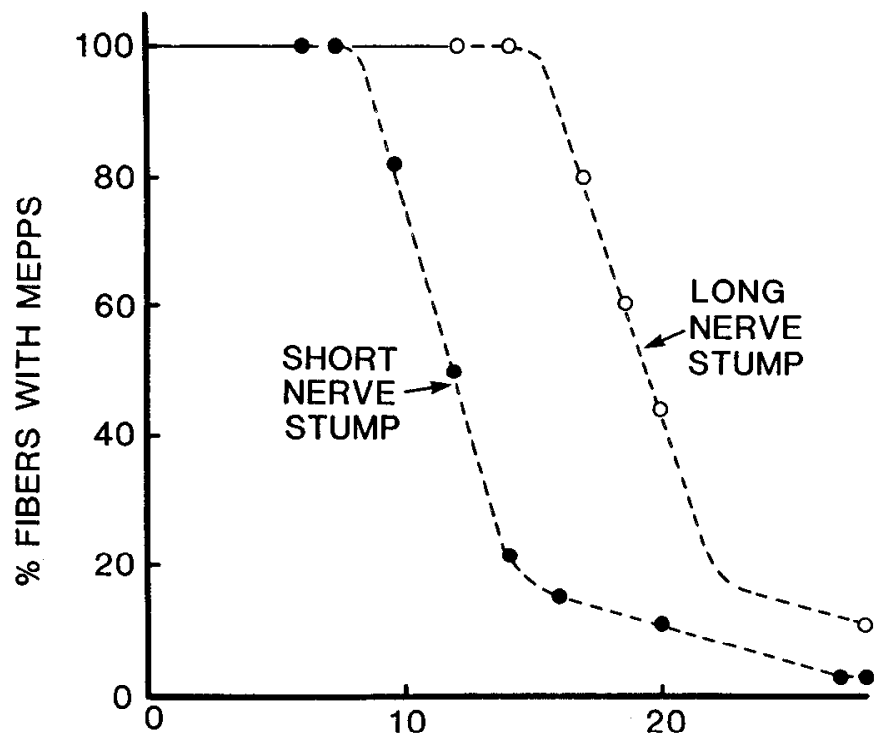

TIME AFTER DENERVATION (HOURS)

Figure 2. Time course of denervation-induced MEPP failure in vitro Diaphragms were incubated in vitro as described under "Materials and Methods." The percentage of fibers with MEPPs in diaphragms with short nerve stump (O) or long nerve stump (O) is plotted as a function of time after denervation. MEPP failure showed a $t^{1 / 2}$ of $12 \mathrm{hr}$ in $-\mathrm{N}$ strips and 19.5 $\mathrm{hr}$ in $+\mathrm{N}$ strips. Each data point is the average of 60 fibers from three preparations.

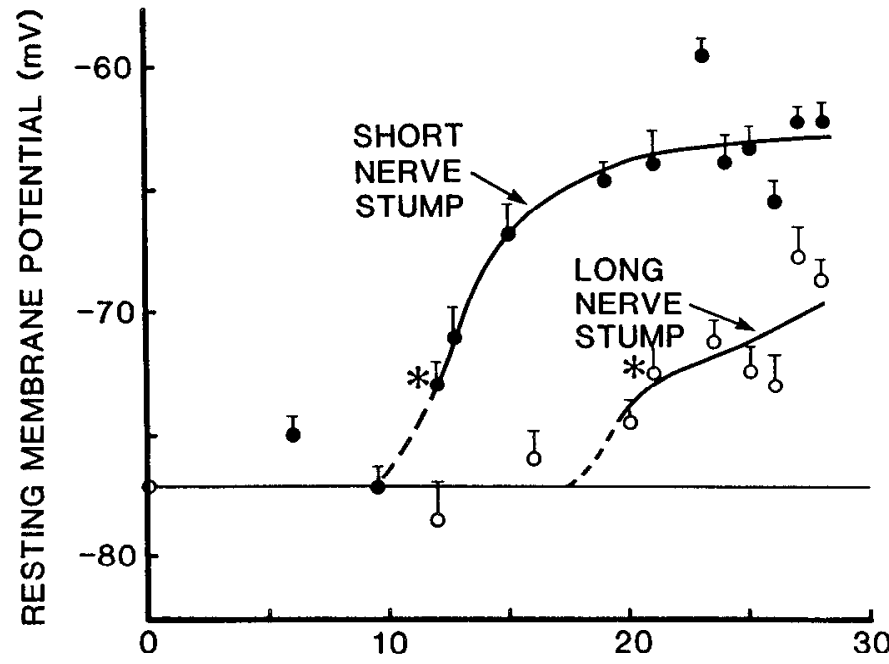

TIME AFTER DENERVATION (HOURS)

Figure 3. Time course of denervation-induced depolarization of RMP in vitro. The RMP of diaphragms with short nerve stump (O) and long nerve stump $(O)$ are plotted as a function of time. Each data point is the average of 60 fibers from three preparations. * Pooled data at these and later time points show RMP significantly different $(p<0.01)$ from control (fresh dissected or preceding times in vitro).

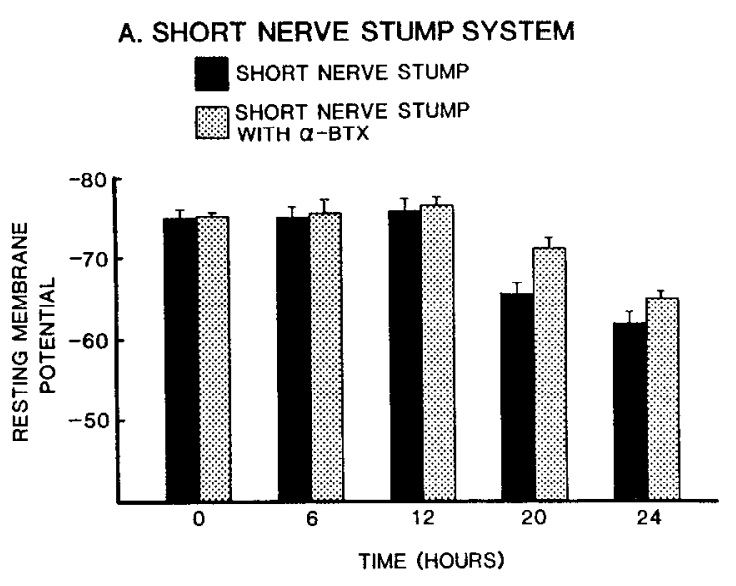

B. LONG NERVE STUMP SYSTEM

LONG NERVE STUMP LONG NERVE STUMP $+\alpha B T X(*)$ OR CURARE $(0)$

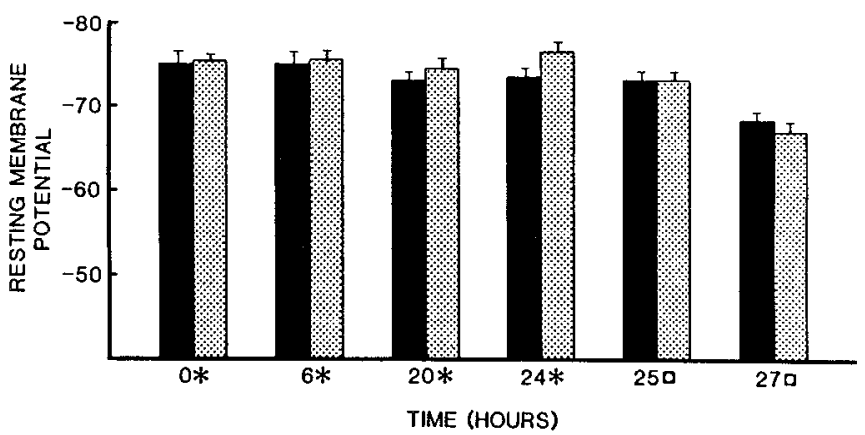

Figure 4. Effect of cholinergic blockers on denervation-induced depolarization of membrane potential. $\alpha$-Bungarotoxin $(0.6 \mu \mathrm{M})$ or curare $(4 \mu \mathrm{M})$ were present throughout the incubation period. The RMPs of diaphragms with short nerve stump $(A)$ or long nerve stump $(B)$ are plotted as a function of time after denervation. Solid bars, control; hatched bars, $\alpha$-BTX (*) or curare $(\mathbf{0})$. 


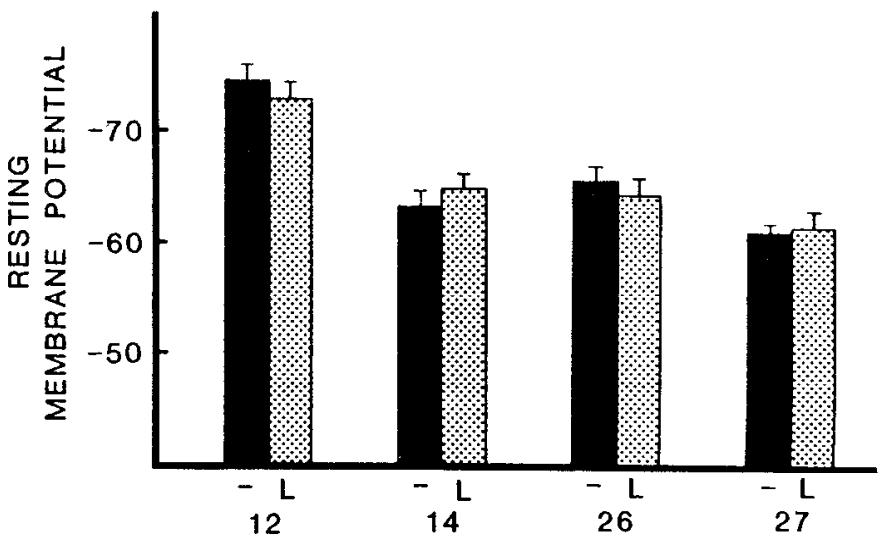

TIME (HOURS)

Figure 5. Effect of the protease inhibitor leupeptin on RMP after denervation. Leupeptin (4 or $20 \mu \mathrm{M}$ ) was present throughout the incubation period. The RMPs are plotted as a function of time. Solid bars represent short nerve stump; hatched bars represent short nerve stump with leupeptin. Each data point is the average of 20 fibers from one preparation.

Card, 1977; Komatsu et al., 1984), it was of interest to determine whether this type of nicotinic proteolytic mechanism played a role in denervation-induced membrane depolarization.

\section{Materials and Methods}

Materials. $\alpha$-Bungarotoxin was obtained from Miami Serpentarium, and $d$ tubocurarine and leupeptin were from Sigma Chemical Co. Male SpragueDawley rats weighing 90 to $120 \mathrm{gm}$ were obtained from Hilltop Laboratory Animals, Inc.

In vivo denervation. Rats were anesthetized with ether and a small incision was made in the peritoneal wall along the lower border of the left rib cage, exposing the abdominal surface of the diaphragm. The intradiaphragmatic right branch of the left phrenic nerve was sectioned about $1 \mathrm{~mm}$ beyond its entrance into the diaphragm. The abdominal wall was then sutured, and the rat was allowed to recover. The muscle strip innervated by the intact left branch served as an internal sham control. At appropriate times, the diaphragms were removed and electrophysiologic investigation was performed, as described below.

Organ culture. Left hemidiaphragms were removed within $5 \mathrm{~min}$ after doath from rats killed by head concussion. The muscle was then cut into paired strips, one with and one without a $2-\mathrm{cm}$ phrenic nerve stump (" $+\mathrm{N}$ " and " $-N$ ", respectively), and was incubated as previously described (Robbins, 1981; Olek and Robbins, 1981). Briefly, the strips were placed for various times in organ culture $\left(36^{\circ} \mathrm{C}\right.$, oxygenated Medium 199) with continuous rocking $(1.5 \mathrm{~min}$ atmosphere, $0.5 \mathrm{~min}$ immersed). In the indicated experıments, a nicotınic blocker, $\alpha$-BTX $(0.6 \mu \mathrm{M})$ or $\alpha$-tubocurarine $(4 \mu \mathrm{M})$, or the protease inhibitor leupeptin $(4$ or $20 \mu \mathrm{M}$ ) was added to the medium. Upon completion of the incubation period, the diaphragm strips were placed in oxygenated Krebs solution $\left(25^{\circ} \mathrm{C}\right)$, and the RMPS and MEPPSs of surface muscle fibers were recorded, using glass microelectrodes filled with $3 \mathrm{M} \mathrm{KCl}$ (15 megohms DC resistance, tip potential less than 2 to $3 \mathrm{mV}$ ) as previously described (Robbins, 1981). At the conclusion of the electrophysiology, some of the ("interim") preparations were reincubated and reused for electrophysiological measurements at later time points. RMPs at each time represent the average of two interim and two terminal recordings. There was no statistical difference between observations in muscles from terminal and repeatedly sampled organ cultures

\section{Results}

Denervation-induced RMP depolarization in vivo. In hemidiaphragm denervated in vivo with a short nerve stump (2 to $7 \mathrm{~mm})$, MEPPS disappeared between 8 and $10 \mathrm{hr}$ after denervation (with half-life of $9 \mathrm{hr}$ ), and the depolarization of the RMP occurred several hours later (Fig. 1). In the control portions of diaphragms, MEPPs were maintained in $100 \%$ of the muscle fibers, and RMP was unchanged. These results are similar to those reported in extensor digilorium longus (EDL) and soleus muscles (Card, 1977).

Time course of denervation-induced MEPP failure in vitro. Rat left hemidiaphragms with short or with long nerve stumps were incubated for various times, and the percentage of fibers with MEPPs was determined upon termination of the incubation (Fig. 2). Following denervation, MEPPs disappeared with a half-time of $12 \mathrm{hr}$ in $-\mathrm{N}$ muscles (with 0- to 5-mm nerve stump) and $19.5 \mathrm{hr}$ in $+\mathrm{N}$ muscles with a 2-cm stump. Thus, as reported previously (Robbins, 1981), the denervation-dependent disappearance of MEPPs as well as the nerve stump effect delaying MEPP failure occurred in vitro as it does in vivo (Miledi and Slater, 1970) and with similar time course.

Time course of denervation-induced depolarization of RMP in vitro. Rat left hemidiaphragms were maintained in organ culture for various periods of time after the denervation entailed in removing the muscles from the animal. Resting membrane potentials, as determined upon termination of the culture, declined between 12 and $19.5 \mathrm{hr}$ after denervation in $-\mathrm{N}$ muscles and between 20 and $27 \mathrm{hr}$ in $+\mathrm{N}$ muscles (Fig. 3). The in vitro delay of RMP depolarization produced by the nerve stump closely resembles the previously described in vivo nerve stump effect on RMP (Card, 1977).

Effect of cholinergic blockers on denervation-induced depolarization of membrane potential. The cholinergic blockers, $\alpha$-BTX $(0.6$ $\mu \mathrm{M})$ or curare ( $d$-tubocurarine, $4 \mu \mathrm{M})$, produce "instant cholinergic denervation" and, thus, should accelerate the onset of denervation effects if nicotinic ACh transmission plays a role in maintenance of normal RMP. The most critical time points for observations of this acceleration are those prior to the time of expected membrane depolarization (i.e., before 12 and $22 \mathrm{hr}$ in short- or long-stump preparations, respectively) and also those still earlier times when ACh might still be released at denervated endplates (see "Discussion"). Therefore, all studies were focused only on time periods up to about $27 \mathrm{hr}$ after denervation. Nicotinic transmission in the treated muscles was blocked by $\alpha$-BTX or curare from the initiation of the culture: no MEPPs, indirect contractions, or endplate potentials could be recorded in freshly dissected blocked preparations. An in vitro analysis was essential since, in vivo, $\alpha$-BTX both wears off and cannot be administered safely at sufficiently high concentration to produce complete and continuous inhibition of AChR

The major findings (Fig. 4) were that $\alpha$-BTX did not accelerate RMP depolarization in denervated muscle fibers with short nerve stump, nor did $\alpha$-BTX or curare accelerate the depolarization of muscle fibers supplied by a long nerve stump. Since inhibitors of nicotinic receptors did not alter the delaying effect of the nerve stump on RMP decline, the nerve stump effect on RMP cannot result from a nicotinic cholinergic mechanism. There was only one statistically significant difference between blocked and denervated muscles, namely, at $20 \mathrm{hr}$ in $-\mathrm{N}$ cultures (Fig. 4A). However, the absolute difference was only $5 \mathrm{mV}$ and the blocked muscles had greater membrante potentials (i.e., the opposite sign from thal predicted if ACh release were important in maintenance of RMP). Furthermore, in $+\mathrm{N}$ preparations, which should even more clearly demonstrate an acceleratory effect of nicotinic blockers on membrane depolarization, there were no differences with or without the nicotinic blockers even when (at $27 \mathrm{hr}$ ), RMP began to diminish (Fig. 4B).

Effect of the protease inhibitor, leupeptin, on resting potential and MEPP alteration. If leupeptin-sensitive proteolytic processes are involved in denervation changes (see the introduction), leupeptin should prevent or delay such changes. Therefore, left hemidiaphragm strips were incubated in the presence or absence of leupeptin $(4 \mu \mathrm{M}$, or $20 \mu \mathrm{M})$ for $12,14,26$, or $27 \mathrm{hr}$, and RMP and MEPPs were recorded. The presence of the leupeptin in the medium did not prevent or delay presynaptic MEPP failure or the denervationinduced membrane depolarization (Fig. 5). Thus, cellular processes underlying membrane depolarization do not include a leupeptinsensitive protease component

\section{Discussion}

As explained in the introduction, it was essential in these experiments to ensure that nicotinic receptors were entirely blocked throughout the post-denervation period in which ACh release de- 
clined and membrane depolarization began. Since an organ culture system was used to attain this objective, it is also important to establish that this system sufficiently reproduces post-denervation events in vivo. This was shown here and previously for several parameters.

In organ culture, the time course of the disappearance of MEPPs and of the depolarization of the RMP closely resembled those reported for the corresponding changes observed in vivo (Miledi and Slater, 1970; Card, 1977). Other dencrvation phenomena that showed similar time courses in vivo and in vitro included increase of extrajunctional ACh receptor (Olek and Robbins, 1981) and increase of glucose 6-phosphate dehydrogenase (G6PD) activity (Robbins, 1981). Finalisy, delay of onset of denervation phenomenon by the presence of a long nerve stump was also reproduced in vitro in respect to timing of MEPP failure, depolarization of membrane potential, and increase of G6PD (present study; Robbins, 1981). In all of these cases of close similarity between in vivo and in vitro phenomena, the only difference in the two conditions was a shift of 3 to $4 \mathrm{hr}$ in time of appearance of certain denervation phenomena. Small temperature differences $\left(36^{\circ} \mathrm{C}\right.$ in vitro versus $38^{\circ} \mathrm{C}$ in vivo) and an initial equilibration period could account for these minor disparities. Furthermore, the maintenance of normal RMP in muscles with long nerve stumps argues for the viability of muscle under these in vitro conditions. Thus, there is strong evidence that this system is suitable for in vitro study of neurotrophic regulation of muscle RMP.

Our experiments were designed to test the hypothesis that $\mathrm{ACh}$ released from nerve terminals is a neurotrophic factor producing its effect through nicotinic receptors (Drachman et al., 1982). The present experiments with nicotinic blockers can only be evaluated after discussing the time course of ACh release after denervation. Acetylcholine transmission (spontaneous quantal release) in muscles with short or long nerve stumps failed with half-lives of 12 and $20 \mathrm{hr}$ after denervation, respectively, in general agreement with previous and the current reports based on in vivo denervations (Slater, 1966; Redfern and Thesleff, 1971; Card, 1977; Drachman et al., 1982). Also, non-quantal $\mathrm{ACh}$ release, which may comprise most spontaneous $\mathrm{ACh}$ release, declines significantly by $11 \mathrm{hr}$ (to $75 \%$ ) and to $50 \%$ of control by $18 \mathrm{hr}$ after denervation (Linden et al., 1983); choline acetyltransferase follows a similar time course (Miledi et al., 1981, 1982). If quantal or non-quantal ACh were a major trophic influence preventing depolarization, then nicotinic blockers should have accelerated the depolarization of RMP by 8 to $11 \mathrm{hr}$, an effect which would have been readily detected in our system. Yet, blockade of nicotinic transmission did not advance the time at which the RMP declined in muscles with either short or long nerve stumps. Hence, the neuronal regulation of RMP is not entirely explicable by a nicotinic mechanism

The result of the nerve stump experiments combined with those with nicotinic blockers further strengthen the case against $\mathrm{ACh}$ as a critical trophic factor. First, if any form of ACh release (but especially spontaneous quantal or non-quantal $A C h$ ) were involved in the delaying effect of the nerve stump on RMP depolarization, it would be necessary that this influence persist for $8 \mathrm{hr}$ longer in the case of the long nerve stump preparation. Yet, even in muscles cultured with long nerve stumps, the timing of membrane depolarization was unaffected by the continuous presence of nicotinic blockers. Second, it might be proposed that nicotinic blockers had no accelerating action on membrane depolarization solely because a minimum time (equal to that obtained with a short nerve stump) is required for development of the denervation effect. However, the delay of the effects of denervation by the presence of a nervo stump and the lack of effect of nicotinic blockers in this condition as well rules out this possibility.

Our results are at odds with those of Bray et al. (1982), in which rat diaphragm placed in organ culture showed a nerve stumpdependent depolarization after only $3 \mathrm{hr}$ in culturc, and in which cholinergic blockers accelerated depolarization in preparations with longer nerve stumps. First, a depolarization in 3 or $6 \mathrm{hr}$ has not been observed in vivo by most workers (Miledi and Slater, 1970; Card, 1977; Drachman et al., 1982; present report). In our case, we used a transabdominal in vivo denervation to mimic closely the short length of nerve stump in the organ-cultured muscles. Second, as noted above, the time courses of the changes seen in our preparation in vitro closely conform to those of a number of denervation events in vivo, whereas this is not clear in the cited work (and certainly not so for membrane depolarization). Therefore, we suggest that the unusually rapid membrane depolarization noted by Bray et al. (1982) may reflect peculiar culture conditions (e.g., insufficient oxygenation, an effect of Trowell's T-8 medium in which spontaneous contractions occur in the first hours of incubation (unpublished observations)), or conditions in which a nicotinic proteolytic mechanism is provoked.

In conclusion, the studies described in this paper provide evidence for a neurotrophic influence independent of cholinergic transmission. Thus, as an overview, the neuronal influence on muscle appears to consist of three functions: (1) stimulation of muscle activity (Drachman and Witzke, 1972; L $\phi$ mo and Rosenthal, 1972; Shainberg and Burstein, 1976; Hall and Reiness, 1977; Betz and Changeux, 1979), (2) nicotinic influence as supported by the tetrodotoxin experiments (Lavoie et al., 1976; Pestronk et al., 1976) combined with results of experiments with $\alpha$-BTX (Drachman et al., 1982), and (3) a nonactivity-dependent non-nicotinic function, as indicated by the present study (see also Tobias and Masukawa, 1983, for non-activity effects). Our data provide no indication of the nature of this latter function. A non-nicotinic trophic action of $\mathrm{ACh}$, trophic factor(s) other than $\mathrm{ACh}$, or an interaction between nerve, muscle, and synaptic matrix are all possible trophic mechanisms consistent with our data.

Another hypothesis to explain the third mechanism would have to do with nerve terminal degeneration products initiating the early response of denervation. In this event, leupeptin, a lysosomal protease inhibitor, might delay denervation effects or delay the onset of a denervation phenomenon such as resting membrane depolarization. However, leupeptin did not delay MEPP failure or alter denervation-induced changes in RMP. Although leupeptin apparently does prevent or delay loss of actylcholinesterase activity (Fernandez and Duell, 1980) and muscle atrophy (Libby and Goldberg, 1980) after denervation, the neural regulation of RMP may have a different mechanism.

\section{References}

Albuquerque, E. X., F. T. Schuh, and F. C. Kauffman (1971) Early membrane depolarization of the fast mammalian muscle after denervation. Pflugers Arch. 328: 36-50.

Betz, H., and J. P. Changeux (1979) Regulation of muscle acetylcholine receptor synthesis in vitro by cyclic nucleotide derivatives. Nature 278 : $749-752$.

Bray, J. J., J. W. Forrest, and J. I. Hubbard (1982) Evidence for the role of non quantal acetylcholine in the maintenance of the membrane potential of rat skeletal muscle. J. Physiol. (Lond.) 326: 285-296.

Card, D. J. (1977) Denervation: Sequence of nouromuscular degenerative changes in rats and the effect of stimulation. Exp. Neurol. 54: 251-265.

Cater, J. L., and S. Brimijoin (1981) Effects of acute and chronic denervation on release of acelylcholinesterase and its molecular forms in rat diaphragms. J. Neurochem. 36: 1018-1025.

Cohen, S. A., and J. D. Fishbach (1973) Regulation of muscle acetylcholine sensitivity by muscle activity in cell culture. Science 181: /6-/8.

Drachman, D. B., and F. Witzke (1972) Trophic regulation of acetylcholine sensitivity of muscle: Effect of electrical stimulation. Science 176: 514516

Drachman, D. B., E. F. Stanley, A. Pestronk, J. W. Griffin, and D. L. Price (1982) Neurotrophic regulation of two properties of skeletal muscle by impulse-dependent and spontaneous acetylcholine transmission. J. Neurosci. 2: 232-243

Fambrough, D. M. (1979) Control of acetylcholine receptors in skeletal muscle. Physiol. Rev. 59: 165-227.

Fernandez, H. L., and M. J. Duell (1980) Protease inhibitors reduce effects of denervation on muscle endplate acetylcholinesterase. J. Neurochem. 35: $1166-1171$. 
Gutman, E. (1976) Neurotrophic relations. Annu. Rev. Physiol. 38: 177-216. Hall, Z. W., and C. G. Reiness (1977) Electrical stimulation of denervated muscle reduces incorporation of methionine into the ACh receptor. Nature 268: 655-657.

Harris, A. J. (1974) Inductive functions of the nervous system. Annu. Rev. Physiol. 36: 251-305

Komatsu, K., K. Uchida, and S. Satoh (1984) Neurotrophic influences are not affected by miniature end-plate potentials. Exp. Neurol. 83: 33-41.

Lavoie, P. A., B. Collier, and H. Tenenhouse (1976) Comparison of $\alpha$ bungarotoxin binding to skeletal muscles after inactivity or denervation. Nature 260: 349-350

Leonard, J. P., and M. M. Salpeter (1979) Agonist induced myopathy at the neuromuscular junction is mediated by calcium. J. Cell Biol. 82: 811-819.

Libby, P., and A. L. Goldberg (1980) Effects of chymostatin and other proteinase inhibitors on protein breakdown and proteolytic activities in muscle. Biochem. J. 188: 213-220.

Linden, D. C., M. W. Newton, A. D. Grinnell, and D. J. Jenden (1983) Rapid decline in acetylcholine release and content of rat extensor digitorium longus muscle after denervation. Exp. Neurol. 81: 613-626.

L $\phi$ mo, T., and J. Rosenthal (1972) Control of ACh sensitivity by muscle activity in the rat. J. Physiol. (Lond.) 221: 493-513.

Miledi, R., and C. R. Slater (1970) On the degeneration of rat neuromuscular junctions after nerve section. J. Physiol. (Lond.) 207: 507-528.

Miledi, R., P. C. Molenaar, and R. L. Polak (1981) Early effects of denervation on acetylcholine and choline acetyltransferase in skeletal muscle. Adv. Behav. Biol. 25: 205-214.
Miledi, R. P. C. Molenaar, R. L. Polak, J. W. Tas, and T. Van Der Laaken (1982) Neural and non neural acetylcholine in the rat diaphragm. Proc. R. Soc. Lond. (Biol.) 214: 153-168.

Olek, A. J., and N. Robbins (1981) A nerve stump dependent appearance of junctional and extrajunctional acetylcholine receptors in organ culture. Neuroscience 6: 1771-1782.

Pestronk, A., D. B. Drachman, and J. W. Griffin (1976) Effect of muscle disuse on acetylcholine receptors. Nature 260: 252-253.

Purves, D. (1976) Long term regulation in the vertebrate peripheral nervous system. Int. Rev. Physiol. 10: 125-178.

Redfern, P., and S. Thesleff (1971) Action potential generation in denervated rat skeletal muscle. II. The action of tetrodotoxin. Acta Physiol. Scand. 82: $70-78$.

Robbins, N. (1981) Neurotrophic regulation of rat muscle glucose-6-phosphate dehydrogenase in vitro. Brain Res. 225: 387-399

Rochel, S., and N. Robbins (1984) Receptor occupancy and turnover in cholinergic transmission. Soc. Neurosci. Abstr. 10: 276.

Shainberg, A., and M. Burstein (1976) Decrease of acetylcholine receptor synthesis in muscle cultures by electrical stimulation. Nature 264: 358367

Slater, C. R. (1966) Studies on denervated mammalian skeletal muscle. Ph.D. thesis, University College, London,

Tobias, G. S., and L. M. Masukawa (1983) A comparison of nerve transection and chronic application of $\beta$-bungarotoxin on acetylcholine receptor distribution and other nerve-muscle properties. Exp. Neurol. 79: 583-600. 